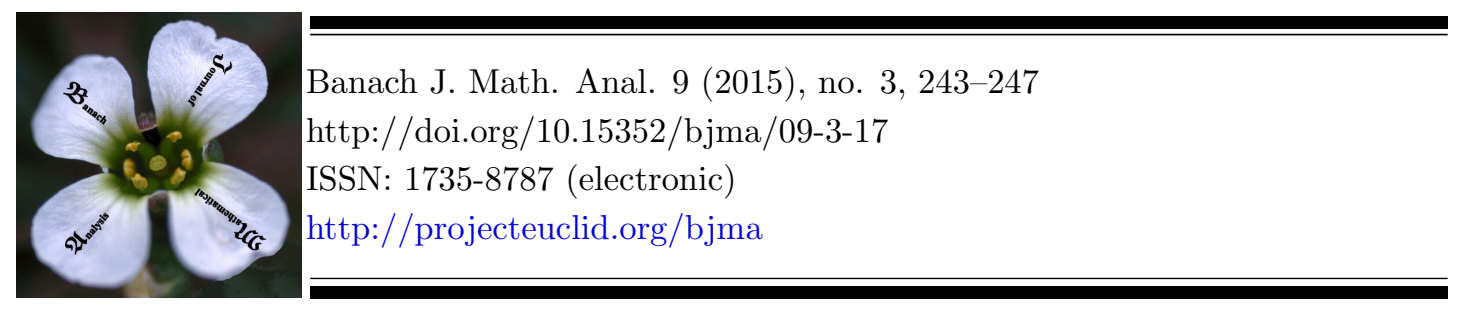

\title{
DETERMINANTAL INEQUALITIES FOR HADAMARD PRODUCT OF $M$-MATRICES AND INVERSE $M$-MATRICES
}

\author{
XIAOHUI FU $\mathrm{F}^{1,2^{*}}$, CHUANJIANG HE ${ }^{1}$ \\ Communicated by F. Zhang
}

\begin{abstract}
In this paper, we generalize some determinantal inequalities for Hadamard product of $M$-matrices and inverse $M$-matrices obtained by Chen [Linear Algebra Appl. 426 (2007), no. 2-3, 610-618.].
\end{abstract}

\section{INTRODUCTION}

Let $C^{m \times n}\left(R^{m \times n}\right)$ be the set of all complex (real) matrices. A real matrix is nonnegative if every entry is nonnegative. For two $m \times n$ matrices $A=\left(a_{i j}\right), B=$ $\left(b_{i j}\right) \in R^{m \times n}$, we write $A \geq B$ if $A-B$ is nonnegative. The Hadamard product of $A=\left(a_{i j}\right), B=\left(b_{i j}\right) \in C^{m \times n}$ is $A \circ B=\left(a_{i j} b_{i j}\right) \in C^{m \times n}$.

Suppose that $N=\{1,2, \ldots, n\}$ and the index set $\alpha=\left\{i_{1}, i_{2}, \ldots, i_{s}\right\} \subseteq N$. The principal submatrix of an $n \times n$ matrix $A$ including rows and columns $i_{1}, i_{2}, \ldots, i_{s}$ is denoted by $A\left[i_{1}, i_{2}, \ldots, i_{s}\right]$ or $A[\alpha]$. In particular, the $k \times k$ leading principal submatrix of $A$ is denoted by $A_{k}=A[1,2, \ldots, k](k \in N)$ and we set $A(k)=$ $A[N \backslash\{k\}]$.

Let $Z^{n \times n}=\left\{A=\left(a_{i j}\right) \in R^{n \times n}: a_{i j} \leq 0, i \neq j, i, j \in N\right\} . A \in Z^{n \times n}$ is called an $M$-matrix if it is nonsingular and its inverse is a nonnegative matrix. We denote by $M_{n}$ the class of all $n \times n M$-matrices. If $A^{-1} \in M_{n}$, then we say $A$ is an inverse $M$-matrix. The class of $n \times n$ inverse $M$-matrices is denoted by $M_{n}^{-1}$. Clearly, if $A \in M_{n}^{-1}$, then $A$ is necessarily nonnegative.

Date: Received: Oct. 9, 2014; Revised: Nov. 3, 2014; Accepted: Nov. 10, 2014.

* Corresponding author.

2010 Mathematics Subject Classification. Primary 15A15; Secondary 47A63.

Key words and phrases. Hadamard product, determinantal inequality, $M$-matrix, inverse $M$-matrix. 
Chen [1, Theorem 3.3] proved the following determinantal inequalities for the Hadamard product of an $M$-matrix and an inverse $M$-matrix: if $A=\left(a_{i j}\right) \in M_{n}$, $B=\left(b_{i j}\right) \in M_{n}^{-1}$, then $A \circ B \in M_{n}$, and for any permutation $i_{1}, i_{2}, \ldots, i_{n}$ of $N$, the following inequalities hold:

$$
\begin{aligned}
\operatorname{det}(A \circ B) \geq & \operatorname{det}(A B) \times \\
& \prod_{s=2}^{n}\left(\frac{a_{i_{s} i_{s}} \operatorname{det} A\left[i_{1}, \ldots, i_{s-1}\right]}{\operatorname{det} A\left[i_{1}, \ldots, i_{s-1}, i_{s}\right]}+\frac{b_{i_{s} i_{s}} \operatorname{det} B\left[i_{1}, \ldots, i_{s-1}\right]}{\operatorname{det} B\left[i_{1}, \ldots, i_{s-1}, i_{s}\right]}-1\right),
\end{aligned}
$$

and

$$
\begin{aligned}
\operatorname{det}(A \circ B) \leq & \left(\prod_{i=1}^{n} a_{i i} b_{i i}\right) \times \\
& \prod_{s=2}^{n}\left(1-\frac{\left|a_{i_{1} i_{2}} \cdots a_{i_{s-1} i_{s}} a_{i_{s} i_{1}}\right|}{a_{i_{1} i_{1}} a_{i_{2} i_{2}} \cdots a_{i_{s} i_{s}}} \cdot \frac{b_{i_{1} i_{2}} \cdots b_{i_{s-1} i_{s}} b_{i_{s} i_{1}}}{b_{i_{1} i_{1}} b_{i_{2} i_{2}} \cdots b_{i_{s} i_{s}}}\right) .
\end{aligned}
$$

Very recently, Lin [3] proved that for the block positive definite matrices, a similar result to (1.1) holds for a block Hadamard product.

In this paper, we will present two determinantal inequalities for the Hadamard product of an $M$-matrix and some inverse $M$-matrices which are generalizations of (1.1) and (1.2).

\section{MAin Results}

We give some lemmas before we give the main theorem of this paper.

Lemma 2.1. [2, p. 117 and p. 127 Problem 9] If $A=\left(a_{i j}\right) \in M_{n} \cup M_{n}^{-1}$, then for any $i$

$$
\frac{a_{i i} \operatorname{det} A(i)}{\operatorname{det} A} \geq 1
$$

Remark 2.2. Chen [1, Theorem 3.1] proved a stronger result as follows:

If $A=\left(a_{i j}\right) \in M_{n} \cup M_{n}^{-1}$, then for any $\alpha=\left\{i_{1}, i_{2}, \ldots, i_{s}\right\} \subseteq N$, where $i_{1}, i_{2}, \ldots, i_{s}$ are mutually distinct,

$$
0<\frac{\operatorname{det} A}{a_{i_{s} i_{s}} \operatorname{det} A\left(i_{s}\right)} \leq 1-\frac{\left|a_{i_{1} i_{2}} \cdots a_{i_{s-1} i_{s}} a_{i_{s} i_{1}}\right|}{a_{i_{1} i_{1}} a_{i_{2} i_{2}} \cdots a_{i_{s} i_{s}}} .
$$

Lemma 2.3. [1, Theorem 3.3] If $A=\left(a_{i j}\right) \in M_{n}, B=\left(b_{i j}\right) \in M_{n}^{-1}$, then $A \circ B \in M_{n}$.

Now we present our main result:

Theorem 2.4. If $A_{1}=\left(a_{i j}^{(1)}\right) \in M_{n}$ and $A_{i}=\left(a_{i j}^{(i)}\right)(i=2, \ldots, m) \in M_{n}^{-1}$, then the Hadamard product $A_{1} \circ A_{2} \circ \cdots \circ A_{m} \in M_{n}$, and for any permutation $i_{1}, i_{2}, \cdots, i_{n}$ of $N$, the following determinantal inequalities hold:

$$
\begin{aligned}
\operatorname{det}\left(A_{1} \circ A_{2} \circ \cdots \circ A_{m}\right) \geq & \operatorname{det}\left(A_{1} \cdot A_{2} \cdots A_{m}\right) \times \\
& \prod_{s=2}^{n}\left(\sum_{j=1}^{m} \frac{a_{i_{s} i_{s}}^{(j)} \operatorname{det} A_{j}\left[i_{1}, \ldots, i_{s-1}\right]}{\operatorname{det} A_{j}\left[i_{1}, \ldots, i_{s-1}, i_{s}\right]}-(m-1)\right),
\end{aligned}
$$


and

$$
\begin{aligned}
\operatorname{det}\left(A_{1} \circ A_{2} \circ \cdots \circ\right. & \left.A_{m}\right) \leq\left(\prod_{i=1}^{n} a_{i i}^{(1)} a_{i i}^{(2)} \cdots a_{i i}^{(m)}\right) \times \\
& \prod_{s=2}^{n}\left(1-\frac{\left|a_{i_{1} i_{2}}^{(1)} \cdots a_{i_{s-1} i_{s}}^{(1)} a_{i_{s} i_{1}}^{(1)}\right|}{a_{i_{1} i_{1}}^{(1)} a_{i_{2} i_{2}}^{(1)} \cdots a_{i_{s} i_{s}}^{(1)}} \cdot \prod_{j=2}^{m} \frac{a_{i_{1} i_{2}}^{(j)} \cdots a_{i_{s-1} i_{s}}^{(j)} a_{i_{s} i_{1}}^{(j)}}{a_{i_{1} i_{1}}^{(j)} a_{i_{2} i_{2}}^{(j)} \cdots a_{i_{s} i_{s}}^{(j)}}\right) .
\end{aligned}
$$

Proof. By Lemma 2.3, it is straightforward to observe that the Hadamard product $A_{1} \circ A_{2} \circ \cdots \circ A_{m}$ is an $M$-matrix. Use induction on $m$. When $m=2$, the result is (1.1). Suppose that (2.1) holds when $m=k-1$ :

$\operatorname{det}\left(A_{1} \circ \cdots \circ A_{k-1}\right) \geq \operatorname{det}\left(A_{1} \cdots A_{k-1}\right) \times$

$\prod_{s=2}^{n}\left(\frac{a_{i_{s} i_{s}}^{(1)} \operatorname{det} A_{1}\left[i_{1}, \ldots, i_{s-1}\right]}{\operatorname{det} A_{1}\left[i_{1}, \ldots, i_{s-1}, i_{s}\right]}+\cdots+\frac{a_{i_{s} i_{s}}^{(k-1)} \operatorname{det} A_{k-1}\left[i_{1}, \ldots, i_{s-1}\right]}{\operatorname{det} A_{k-1}\left[i_{1}, \ldots, i_{s-1}, i_{s}\right]}-(k-2)\right)$.

When $m=k$, we need to prove the following inequality:

$\operatorname{det}\left(A_{1} \circ A_{2} \circ \cdots \circ A_{k}\right) \geq \operatorname{det}\left(A_{1} \cdot A_{2} \cdots A_{k}\right) \times$

$\prod_{s=2}^{n}\left(\frac{a_{i_{s} i_{s}}^{(1)} \operatorname{det} A_{1}\left[i_{1}, \ldots, i_{s-1}\right]}{\operatorname{det} A_{1}\left[i_{1}, \ldots, i_{s-1}, i_{s}\right]}+\cdots+\frac{a_{i_{s} i_{s}}^{(k)} \operatorname{det} A_{k}\left[i_{1}, \ldots, i_{s-1}\right]}{\operatorname{det} A_{k}\left[i_{1}, \ldots, i_{s-1}, i_{s}\right]}-(k-1)\right)$.

By (1.1), we have

$$
\begin{array}{r}
\operatorname{det}\left(\left(A_{1} \circ \cdots \circ A_{k-1}\right) \circ A_{k}\right) \geq \operatorname{det}\left(\left(A_{1} \circ \cdots \circ A_{k-1}\right) A_{k}\right) \times \\
\prod_{s=2}^{n}\left(\frac{a_{i_{s} i_{s}}^{(1)} \cdots a_{i_{s} i_{s}}^{(k-1)} \operatorname{det}\left(A_{1} \circ \cdots \circ A_{k-1}\right)\left[i_{1}, \ldots, i_{s-1}\right]}{\operatorname{det}\left(A_{1} \circ \cdots \circ A_{k-1}\right)\left[i_{1}, \ldots, i_{s-1}, i_{s}\right]}\right. \\
\left.+\frac{a_{i_{s} i_{s}}^{(k)} \operatorname{det} A_{k}\left[i_{1}, \ldots, i_{s-1}\right]}{\operatorname{det} A_{k}\left[i_{1}, \ldots, i_{s-1}, i_{s}\right]}-1\right) .
\end{array}
$$

By the induction hypothesis, it follows

$$
\begin{aligned}
& \operatorname{det}\left(\left(A_{1} \circ \cdots \circ A_{k-1}\right) A_{k}\right) \times \\
& \prod_{s=2}^{n}\left(\frac{a_{i_{s} i_{s}}^{(1)} \cdots a_{i_{s} i_{s}}^{(k-1)} \operatorname{det}\left(A_{1} \circ \cdots A_{k-1}\right)\left[i_{1}, \ldots, i_{s-1}\right]}{\operatorname{det}\left(A_{1} \circ \cdots \circ A_{k-1}\right)\left[i_{1}, \ldots, i_{s-1}, i_{s}\right]}+\frac{a_{i_{s} i_{s}}^{(k)} \operatorname{det} A_{k}\left[i_{1}, \ldots, i_{s-1}\right]}{\operatorname{det} A_{k}\left[i_{1}, \ldots, i_{s-1}, i_{s}\right]}-1\right) \\
& \quad \geq \operatorname{det}\left(A_{1} \cdots A_{k-1}\right) \operatorname{det} A_{k} \times \\
& \prod_{s=2}^{n}\left(\frac{a_{i_{s} i_{s}}^{(1)} \operatorname{det} A_{1}\left[i_{1}, \ldots, i_{s-1}\right]}{\operatorname{det} A_{1}\left[i_{1}, \ldots, i_{s-1}, i_{s}\right]}+\cdots+\frac{a_{i_{s} i_{s}}^{(k-1)} \operatorname{det} A_{k-1}\left[i_{1}, \ldots, i_{s-1}\right]}{\operatorname{det} A_{k-1}\left[i_{1}, \ldots, i_{s-1}, i_{s}\right]}-(k-2)\right) \times \\
& \prod_{s=2}^{n}\left(\frac{a_{i_{s} i_{s}}^{(1)} \cdots a_{i_{s} i_{s}}^{(k-1)} \operatorname{det}\left(A_{1} \circ \cdots \circ A_{k-1}\right)\left[i_{1}, \ldots, i_{s-1}\right]}{\operatorname{det}\left(A_{1} \circ \cdots \circ A_{k-1}\right)\left[i_{1}, \ldots, i_{s-1}, i_{s}\right]}+\frac{a_{i_{s} i_{s}}^{(k)} \operatorname{det} A_{k}\left[i_{1}, \ldots, i_{s-1}\right]}{\operatorname{det} A_{k}\left[i_{1}, \ldots, i_{s-1}, i_{s}\right]}-1\right) .
\end{aligned}
$$

Let

$$
a_{s}=\frac{a_{i_{s} i_{s}}^{(1)} \operatorname{det} A_{1}\left[i_{1}, \ldots, i_{s-1}\right]}{\operatorname{det} A_{1}\left[i_{1}, \ldots, i_{s-1}, i_{s}\right]}+\cdots+\frac{a_{i_{s} i_{s}}^{(k-1)} \operatorname{det} A_{k-1}\left[i_{1}, \ldots, i_{s-1}\right]}{\operatorname{det} A_{k-1}\left[i_{1}, \ldots, i_{s-1}, i_{s}\right]}-(k-2),
$$




$$
\begin{aligned}
b_{s}= & \frac{a_{i_{s} i_{s}}^{(1)} \cdots a_{i_{s} i_{s}}^{(k-1)} \operatorname{det}\left(A_{1} \circ \cdots \circ A_{k-1}\right)\left[i_{1}, \ldots, i_{s-1}\right]}{\operatorname{det}\left(A_{1} \circ \cdots \circ A_{k-1}\right)\left[i_{1}, \ldots, i_{s-1}, i_{s}\right]} \\
& +\frac{a_{i_{s} i_{s}}^{(k)} \operatorname{det} A_{k}\left[i_{1}, \ldots, i_{s-1}\right]}{\operatorname{det} A_{k}\left[i_{1}, \ldots, i_{s-1}, i_{s}\right]}-1 .
\end{aligned}
$$

By Lemma 2.1, we have

$$
\begin{gathered}
\frac{a_{i_{s} i_{s}}^{(l)} \operatorname{det} A_{l}\left[i_{1}, \ldots, i_{s-1}\right]}{\operatorname{det} A_{l}\left[i_{1}, \ldots, i_{s-1}, i_{s}\right]} \geq 1, \quad l=1, \ldots, k, \\
\frac{a_{i_{s} i_{s}}^{(1)} \cdots a_{i_{s} i_{s}}^{(k-1)} \operatorname{det}\left(A_{1} \circ \cdots \circ A_{k-1}\right)\left[i_{1}, \ldots, i_{s-1}\right]}{\operatorname{det}\left(A_{1} \circ \cdots \circ A_{k-1}\right)\left[i_{1}, \ldots, i_{s-1}, i_{s}\right]} \geq 1,
\end{gathered}
$$

and so

$$
a_{s} \geq(k-1)-(k-2)=1, \quad b_{s} \geq 1 .
$$

Using a simple scalar inequality $a b \geq a+b-1$ for $a, b \geq 1$, we obtain the following inequality:

$$
\begin{aligned}
& \operatorname{det}\left(A_{1} \circ \cdots \circ A_{k}\right) \\
& \geq \operatorname{det}\left(A_{1} \cdots A_{k-1}\right) \operatorname{det} A_{k} \times \prod_{s=2}^{n} a_{s} b_{s}(\text { by }(2.3)) \\
& \geq \operatorname{det}\left(A_{1} \cdots A_{k}\right) \times \prod_{s=2}^{n}\left(a_{s}+b_{s}-1\right) \\
& \geq \operatorname{det}\left(A_{1} \cdots A_{k}\right) \times \\
& \prod_{s=2}^{n}\left(\frac{a_{i_{s} i_{s}}^{(1)} \operatorname{det} A_{1}\left[i_{1}, \ldots, i_{s-1}\right]}{\operatorname{det} A_{1}\left[i_{1}, \ldots, i_{s-1}, i_{s}\right]}+\cdots+\frac{a_{s_{s} i_{s}}^{(k)} \operatorname{det} A_{k}\left[i_{1}, \ldots, i_{s-1}\right]}{\operatorname{det} A_{k}\left[i_{1}, \ldots, i_{s-1}, i_{s}\right]}-(k-1)\right)
\end{aligned}
$$

This completes the proof of (2.1).

Next we verify that the inequality (2.2) holds by induction on $m$.

When $m=2$, the inequality (2.2) is (1.2). We assume that it is true when $m=k-1$; namely,

$$
\begin{aligned}
& \operatorname{det}\left(A_{1} \circ A_{2} \circ \cdots \circ A_{k-1}\right) \leq\left(\prod_{i=1}^{n} a_{i i}^{(1)} a_{i i}^{(2)} \cdots a_{i i}^{(k-1)}\right) \times \\
& \prod_{s=2}^{n}\left(1-\frac{\left|a_{i_{1} i_{2}}^{(1)} \cdots a_{i_{s-1} i_{s}}^{(1)} a_{i_{i} i_{1}}^{(1)}\right|}{a_{i_{1} i_{1}}^{(1)} a_{i_{2} i_{2}}^{(1)} \cdots a_{i_{s} i_{s}}^{(1)}} \cdot \frac{a_{i_{1} i_{2}}^{(2)} \cdots a_{i_{s}-1}^{(2)} a_{i_{s} i_{1}}^{(2)}}{a_{i_{1} i_{1}}^{(2)} a_{i_{2} i_{2}}^{(2)} \cdots a_{i_{s} i_{s}}^{(2)}} \cdots \frac{a_{i_{1} i_{2}}^{(k-1)} \cdots a_{i_{s-1} i_{s}}^{(k-1)} a_{i_{s} i_{1}}^{(k-1)}}{a_{i_{1} i_{1}}^{(k-1)} a_{i_{2} i_{2}}^{(k-1)} \cdots a_{i_{s} i_{s}}^{(k-1)}}\right) .
\end{aligned}
$$


When $m=k$, we need to show that

$$
\begin{aligned}
& \operatorname{det}\left(A_{1} \circ A_{2} \circ \cdots \circ A_{k}\right) \leq\left(\prod_{i=1}^{n} a_{i i}^{(1)} a_{i i}^{(2)} \cdots a_{i i}^{(k)}\right) \times \\
& \prod_{s=2}^{n}\left(1-\frac{\left|a_{i_{1} i_{2}}^{(1)} \cdots a_{i_{s-1} i_{s}}^{(1)} a_{i_{s} i_{1}}^{(1)}\right|}{a_{i_{1} i_{1}}^{(1)} a_{i_{2} i_{2}}^{(1)} \cdots a_{i_{s} i_{s}}^{(1)}} \cdot \frac{a_{i_{1} i_{2}}^{(2)} \cdots a_{i_{s-1} i_{s}}^{(2)} a_{i_{s} i_{1}}^{(2)}}{a_{i_{1} i_{1}}^{(2)} a_{i_{2} i_{2}}^{(2)} \cdots a_{i_{s} i_{s}}^{(2)}} \cdots \frac{a_{i_{1} i_{2}}^{(k)} \cdots a_{i_{s-1} i_{s}}^{(k)} a_{i_{s} i_{1}}^{(k)}}{a_{i_{1} i_{1}}^{(k)} a_{i_{2} i_{2}}^{(k)} \cdots a_{i_{s} i_{s}}^{(k)}}\right) .
\end{aligned}
$$

By (1.2) and the induction hypothesis, we have

$$
\begin{aligned}
& \operatorname{det}\left(A_{1} \circ A_{2} \circ \cdots \circ A_{k-1} \circ A_{k}\right)=\operatorname{det}\left(\left(A_{1} \circ A_{2} \circ \cdots \circ A_{k-1}\right) \circ A_{k}\right) \\
& \leq\left(\prod_{i=1}^{n}\left(A_{1} \circ A_{2} \circ \cdots \circ A_{k-1}\right)_{i i} \cdot a_{i i}^{(k)}\right) \times \\
& \prod_{s=2}^{n}\left(1-\frac{\left|\left(a_{i_{1} i_{2}}^{(1)} a_{i_{1} i_{2}}^{(2)} \cdots a_{i_{i} i_{2}}^{(k-1)}\right)\left(a_{i_{2} i_{i}}^{(1)} a_{i_{2} i_{3}}^{(2)} \cdots a_{i_{i} i_{3}}^{(k-1)}\right) \cdots\left(a_{i_{s} i_{1}}^{(1)} a_{i_{s} i_{i}}^{(2)} \cdots a_{i_{s} i_{1}}^{(k-1)}\right)\right|}{\left(a_{i_{1} i_{1}}^{(1)} a_{i_{1} i_{1}}^{(2)} \cdots a_{i_{1} i_{1}}^{(k-1)}\right)\left(a_{i_{2} i_{2}}^{(1)} a_{i_{2} i_{2}}^{(2)} \cdots a_{i_{2} i_{2}}^{(k-1)}\right) \cdots\left(a_{i_{s} i_{s}}^{(1)} a_{i_{s} i_{s}}^{(2)} \cdots a_{i_{s} i_{s}}^{(k-1)}\right)} \cdot \frac{a_{i_{1} i_{2}}^{(k)} \cdots a_{i_{s-1} i_{s}}^{(k)} a_{i_{s} i_{1}}^{(k)}}{a_{i_{1} i_{1}}^{(k)} a_{i_{2} i_{2}}^{(k)} \cdots a_{i_{s} i_{s}}^{(k)}}\right) \\
&=\left(\prod_{i=1}^{n} a_{i i}^{(1)} a_{i i}^{(2)} \cdots a_{i i}^{(k)}\right) \times \\
& \prod_{s=2}^{n}\left(1-\frac{\left|a_{i_{1} i_{2}}^{(1)} \cdots a_{i_{s-1} i_{s}}^{(1)} a_{i_{s} i_{1}}^{(1)}\right|}{a_{i_{1} i_{1}}^{(1)} a_{i_{2} i_{2}}^{(1)} \cdots a_{i_{s} i_{s}}^{(1)}} \cdot \frac{\left.a_{i_{1} i_{2}}^{(2)} \cdots a_{i_{s-1} i_{s}}^{(2)} a_{i_{s} i_{1}}^{(2)} \cdots \frac{a_{i_{1} i_{2}}^{(k)} \cdots a_{i_{s-1} i_{s}}^{(k)} a_{i_{s} i_{1}}^{(k)}}{a_{i_{1} i_{1}}^{(2)} a_{i_{2} i_{2}}^{(2)} \cdots a_{i_{s} i_{s}}^{(2)}}\right) .}{a_{i_{1} i_{1}}^{(k)} a_{i_{2} i_{2}}^{(k)} \cdots a_{i_{s} i_{s}}^{(k)}}\right)
\end{aligned}
$$

This completes the proof of (2.2).

Remark 2.5. The inequalities (2.1) and (2.2) in Theorem 2.4 are generalizations of the inequalities (1.1) and (1.2), respectively.

Acknowledgement. The authors thank the referee and the handling editor for their valuable comments and suggestions which improve the presentation of the paper. The first author was supported by the key project of the applied mathematics of Hainan Normal University and the natural science foundation of Hainan Province (No.114007).

\section{REFERENCES}

1. S. Chen, Inequalities for $M$-matrices and inverse $M$-matrices, Linear Algebra Appl. 426 (2007), no. 2-3, 610-618.

2. R.A. Horn and C.R. Johnson, Topics in Matrix Analysis, Cambridge University Press, New York, 1991.

3. M. Lin, An Oppenheim type inequality for a block Hadamard product, Linear Algebra Appl. 452 (2014), no. 1, 1-6.

1 College of Mathematics and Statistics, Chongqing University, Chongqing, 401331, P. R. China.

E-mail address: chuanjianghe@sina.com

$2^{*}$ School of Mathematics and Statistics, Hainan Normal University, Haikou, 571158, P. R. China.

E-mail address: fxh6662@sina.com 